\title{
8 A dynamic language ability system framework for diagnosing EMI students' readiness of English language ability
}

\author{
Yuyang Cai and Hintat Cheung
}

\section{Introduction}

Internationalization has become a global trend in higher education (Maringe \& Foskett, 2010). Driven by this trend, universities in non-English speaking European and Eastern Asian countries have been providing an increasing number of programs with English as the medium of instruction (EMI) (Dafouz \& Guerrini, 2009; Doiz et al., 2013; Graddol, 2006; Hughes, 2008; Jenkins, 2014; Wächter \& Maiworm, 2014). However, the English proficiency of the local intakes has been found insufficient to sustain meaningful higher education in the context of EMI. These challenges due to language deficiency have been identified in students studying in both Eastern Asian (Evans \& Morrison, 2017; Hu \& Lei, 2014; Toh, 2016) and European countries (Doiz et al., 2012), and have been found to be detrimental to the process of knowledge construction (Toh, 2016).

To understand the language difficulty faced by these EMI students, existing studies have placed exclusive emphasis on analyzing students' needs in different language skills (Berman \& Cheng, 2010; Evans \& Green, 2007; Evans \& Morrison, 2018). Following the same line of thinking, language education and assessment in the context of EMI have been undertaken according to the conventional view that separates language, content (or the meaning carried by the forms of language) (Cai \& Kunnan, 2018; Douglas, 2000), and thinking competence (Cai \& Cheung, 2019). Before students enter their first year of EMI programs, they are usually diagnosed in terms of their linguistic knowledge (vocabulary, grammar, and textual knowledge) as represented in different types of language skills (i.e., listening, reading, speaking, and writing) (Bachman \& Palmer, 1996, 2010). Drawing on different levels of general language proficiency diagnosed, new intakes are then placed into different streams of English enhancement programs (EEP) to be coached intensively in these language skills, usually lasting two academic years (Cai \& Cheung, 2019).

However, language use in an EMI context is more complex than a topup of general language knowledge in addition to subject knowledge and 
other higher-order thinking skills as perceived by language theorists of the conventional view (Bachman \& Palmer, 1996, 2010). Instead, the development of students' language proficiency fulfilling EMI tasks is more than the accumulating of the individual parts of linguistic elements; it is a complex and dynamic process that involves the evolution of language, subject knowledge, and cognitive capacity in an intertwined whole (Han, 2019; Larsen-Freeman, 2019; Larsen-Freeman \& Cameron, 2008; Mele et al., 2010; Spain, 2019).

Our goal in this paper is to propose the framework of the Dynamic Language Ability System (DLAS) for diagnosing students' English language readiness for EMI study and for evaluating the efficiency of EMI programs. Our intention is to understand the extent to which background knowledge (i.e., disciplinary knowledge), language knowledge, and strategic and thinking competence can be represented in the DLAS. Our attention extends to the implications of DLAS for language assessment development for EMI programs and for EMI program evaluation.

\section{Conceptualizations of language ability}

Throughout the history of language research, language proficiency has been referred to as knowledge of different linguistic aspects. Hymes (1972) distinguished between linguistic competence and communicative competence, the former dealing with knowledge of grammar and the latter with appropriateness of language use in specific situations. Canale and Swain (1980) and Canale (1983) posited a concept of communicative competence that contained four components: grammatical or linguistic competence, sociolinguistic competence (appropriateness of language use), discourse competence (for organizing texts), and strategic competence (communication strategies for compensating failure in language use due to insufficient grammatical competence).

Drawing on their antecedents, Bachman (1990) and Bachman and Palmer (1996, 2010) proposed the well-known framework of Communicative Language Ability (CLA). The CLA compresses grammatical competence, textual competence, and pragmatic competence (i.e., sociolinguistic competence) into a single dimension of language competence and sees language proficiency as the interaction between language competence and strategic competence- two core constituents that determine language performance. An essential feature of the CLA is that this framework takes an ambiguous attitude toward the status of subject-matter knowledge.

In the context of Language for Specific Purposes (LSP), scholars such as Douglas (2000) noted the inseparability of subject-matter background knowledge from LSP language performance. He proposed the concept of LSP ability, which emphasizes the interaction between subject-matter background knowledge and other general language proficiency components (i.e., language competence and strategic competence). 
The evolution of language ability theories has clearly expanded beyond a unitary view of linguistic or grammatical knowledge to include textual knowledge, strategic competence, and recently, background knowledge. Meanwhile, thinking or reasoning competence, another critical component, is found scattered in academic writing in language research but rarely formally acknowledged in classic language ability theories. An exception is with Deane et al. (2008). In their development of a new framework for writing assessment, Deane and colleagues emphasize that writing ability involves a complex arrangement of skills including not only language and literacy skills, but also cognitive skills (e.g., document-creation and management skills) and critical thinking skills. The same idea is echoed in Moore (2011), Afshar and Movassagh (2017), Floyd (2011), and most recently, Cai and Cheung (2019).

In short, the evolution of language ability theories is a consequence of the accumulation of new components into the construct of language ability. However, a common deficiency in this theoretical advancement is the inability of these theories to recognize the "interaction" aspect in the language ability system. It is true that models such as CLA and LSP also emphasize the term "interaction," but they are unable to explicate what this interaction means, how it functions, and what implications the interaction, if it exists at all, has for the practice of language assessment and language education.

Another limitation of dominant language ability theory is that relations among different components of language ability are seen as static. Guided by this idea, language assessment programs are usually designed in such a way as to focus on the diagnosis of certain aspects of language knowledge or skills. According to this static diagnosis, students are grouped and trained according to different levels of language proficiency. In this way, students who produce low overall test scores are usually regarded as low-achievers during a fixed period of English language enhancement. Similarly, students diagnosed with deficiency in writing are treated as poor writers and coached in a way that is tailor-made for poor writers. However, different language components do not necessarily develop at the same speed. For the same students, for a certain period of time their vocabulary knowledge may be able to develop faster, whereas their strategic competence may develop slower. For another period of time, their strategic competence may be able to develop faster when their vocabulary knowledge reaches a certain threshold. This dynamic function becomes more complex when considering the interaction between these conventional language ability components and other critical components such as disciplinary knowledge (Cai \& Kunnan, 2018) and thinking skills (Moore, 2011), and between these expanded language ability components and other contextual factors such as academic motivation. This complex, dynamic aspect of language ability, however, is rarely reflected in existing language ability theory. Systems Thinking (ST) (Capra, 1996; Fischer \& Yan, 2002; Larsen-Freeman \& Cameron, 2008; Mele et al., 2010), on the other hand, provides a lens for us to understand these complex and dynamic features potentially underlying the language ability critical for performing EMI tasks. 


\section{Systems Thinking}

As a popular term in human ecology and learning, ST has been defined as "a dynamic framework for describing, assessing, analysing, and explaining how a person and the world function together in human development"(Fischer \& Yan, 2002, p. 3). Bearing similarity with the reductionism that focuses on constitutional components, ST emphasizes that these components are interdependent with each other and with factors in larger outer systems (Ackoff, 1971). Moreover, ST accommodates the changing mechanism within each constitutional component, and their interrelations with each other, and with factors located in larger systems (Ackoff, 1971; Fischer \& Yan, 2002).

ST has become an interdisciplinary theory about nature and human society (Capra, 1996). Under the paradigm of ST, a social phenomenon is not fully comprehensible by being broken into parts; instead, it can only be understood by taking a holistic view (Mele et al., 2010). Following this line of thinking, an education system cannot be fully understood by breaking it down and by measuring and reformulating its parts (Sahlberg, 2012). To better inform the construction of a curriculum, teaching design, and assessment development, educators need to take the perspective of the ST to "see" the whole spectrum of behaviors in the education system (Robinson, 2009, 2015).

To conceptualize education in the paradigm of ST, Jacobson et al. (2016) proposed a set of features falling into two focus areas: (1) collective behaviors of a system, and (2) behaviors of individual agents in a system. The first area contains four features: agents or elements in system (i.e., key components), self-organization, system levels (i.e., Students' Sensitivity to initial conditions), and emergence. The second focus area consists of (1) parallelism (several things occurring simultaneously), (2) conditional actions (the IF and THEN issue), and (3) adaptation and evolution (see Jacobson et al., 2019).

In language research, Larsen-Freeman and colleagues (Larsen-Freeman, 2012, 2019; Larsen-Freeman \& Cameron, 2008) see language learners as agents and propose the Complex Dynamic Systems Theory (CDST) as a perspective to study the language learning process. This CDST contains a relational system that reflects the ST idea that the whole arises from the interactions between the parts (Capra \& Luisi, 2014). The CDST also contains a temporal component, that is, the relational system evolves within key elements (i.e., linguistic elements) as well as outside them, in higher-level factors across time. According to Larsen-Freeman (2019), this space-temporal system evolves in many directions, dependent on its "initial conditions." Within this language system, different components interact nonlinearly; with time this interaction iterates and self-organizes into states with different statuses such as the "attractor state" (stability or trend to stay nearby) or "repellor" (readiness to move away). 


\section{The Dynamic Language Ability System}

Drawing on theories in language assessment and thoughts from ST research, we propose the construct of the Dynamic Language Ability System (DLAS). The DLAS has two subsystems: a space system and a temporal system. The space system has three main features:

1 There are four core components in the DLAS: language competence (i.e., knowledge of language at the lexical, syntactical, and textual levels, and socio-linguistic knowledge), strategic and thinking competence which contains metacognitive (e.g., planning, monitoring, and evaluating) and thinking competence (e.g., analyzing, synthesizing, deductive reasoning, inductive reasoning), and background knowledge (e.g., general world knowledge, cultural knowledge, domain-general and domain-specific content knowledge).

2 These key components interact with each other and beyond with other contextual factors at micro-level (e.g., motivation, emotion, and other individual characteristics), meso-level (e.g., school factors), exo-level (e.g., media) and macro-levels (country culture, ideology, etc.).

3 The interaction between core components and contextual factors are nonlinear. That is, the effects of one core DLAS component on the overall performance of the DLAS are dependent on the nature of other DLAS core components, as well as the nature of factors beyond these core components.

The temporal system mainly captures the dynamic aspect of the DLAS, and has four main features:

1 Parallel pacing: Each core component has the tendency to develop with its own tempo and magnitude. For example, for young learners (agents) of English, their vocabulary knowledge may develop faster than their strategic and thinking competence (e.g., monitoring, deducing, inferring, etc.) during certain time periods; whereas when the agent becomes more mature, the development in strategic and thinking competence may become faster.

2 Dynamic interdependence: On the condition of parallel tempo, the development of each core component may depend on the development of another component. For instance, the development of thinking competence may have to depend on the development of the agent's vocabulary knowledge and world knowledge.

3 Bipolarity: Bipolarity is the direction of both parallel pacing and dynamic interdependence. For parallel pacing, the development may either tend to increase or decrease. For dynamic interdependence, the interference of one parallel pacing may either increase or decrease the parallel pacing 
of another core component, or the parallel pacing of other contextual factors located across the micro-, meso-, exo- and macro-levels.

4 Fluctuation: The parallel pacing and dynamic interdependence may coexist in a non-monotonous way. For the parallel pacing, the trajectory may display in an up-down pattern, or in a more complex pattern such as up-down-up.

\section{Applying DLAS for language assessment in EMI context}

\section{Principles of DLAS development}

An essential principle of DLAS is that language-oriented EMI assessments should not only provide valid information about the key components involved during knowledge construction, but also about the dynamic evolution of each component and the evolving relations among these components. DLAS offers a mechanism for achieving these twofold goals.

First, a DLAS program can be developed to support claims like those required by conventional language assessments. The total score of a DLAS is the reflection of the overall achievement in one or more core components of the DLAS, as required by EMI teachers for evaluating the learning outcomes of the students or by educational administrators for evaluating the efficiency of the EMI program. A common practice for using total test scores would be to set up a cut-off point as a reflection of the desired competence threshold (Hambleton \& Pitoniak, 2006). However, in the scenario of DLAS, a more subtle treatment is desirable, by zooming into the subscores underlying the total scores, the subscores that represent language competence, strategic and thinking competence, and background knowledge. Of special note is that language assessments in EMI contexts are rarely designed based on such theoretical grounding and therefore, cannot offer subtle diagnostic information regarding students' competence in the core DLAS components. Nor is this analytical approach toward task performance evaluation implemented when EMI subject or language teachers mark their students' task performance.

Another key principle of the DLAS is its focus on the evolutionary trajectories of core components and the relationships within the evolution of each trajectory. DLAS assessment programs of this type repeatedly measure different core components of the DLAS. This repetition allows people to measure where students are on one or more spots on the learning roadmaps, and to explore the change of each component and the relationships between these changes. The purpose of providing such information is to give both classroom teachers and students additional information regarding the weaknesses and strengths of students in different aspects and at different learning stages, an idea best regarded in formative assessment (Scriven, 1967); and to track the change or stability of these weaknesses and strengths across time, a key function of the so-called ipsative assessment (Hughes, 2011, 2014). 
These key features need to be instantiated for each practice of the DLAS language assessment program and EMI classrooms closely tied to the purpose of the DLAS assessment. For the development of language assessments in EMI contexts, a needs analysis must be conducted to understand the demands of various EMI curricula in terms of the key components of the DLAS. Here the structure of the DLAS can function as a model for assessment developers to analyze the curriculum descriptions, descriptions of tasks, oral, written work, or physical or virtual artifacts produced by EMI students for the sake of fulfilling the requirements of the EMI programs (e.g., Cai \& Cheung, 2019). Beyond ensuring that each core component can be identified in these materials, it is important for test developers to identify the combinatorial patterns of these core components in the materials, and to trace the transition of these various combinations across different stages of study (Nesi \& Gardner, 2012).

This transitional structure of DLAS components can be converted into a formal assessment design by mapping the different emphases of required componential competences (i.e., language competence, background knowledge, and strategic and thinking competence) during different stages of EMI studies. More ideally, each of the componential competences can be further decomposed to different extents to create a progressive map. During test construction, general item writing guidelines can be produced to guide item writers to create items that link to the progress variables associated with each phase and that jointly characterize progress on a learning map for DLAS. Figure 8.1 presents the blue print for developing this progressive assessment of DLAS for EMI contexts.

\section{Validity of the DLAS}

In educational measurement, validity has been touted as the core quality of any educational assessment (Messick, 1989). Validation refers to the process of validating the model, centering on assuring claims that the model is consistent with the educational reality that it purports to represent (WolfBranigin, 2013). The validation of a language assessment program based on the DLAS model generally involves the following aspects: componentiality, interaction, progression, transition validity, and bipolarity.

In componentiality validation, the aim is typically to examine the extents to which performance is dependent on the aspects of DLAS componential competences (i.e., language competence, disciplinary knowledge, and strategic and thinking competence). There are multiple ways of generating evidence for componential validity. A straightforward way is to conduct quantitative studies to examine the extent to which a certain componential competence contributes to test takers' performances on the assessment tasks. Methodologically, this approach is no different from validation studies focusing on construct representation (Messick, 1989). However, there is a substantive difference between such studies and componential validity. Simply 


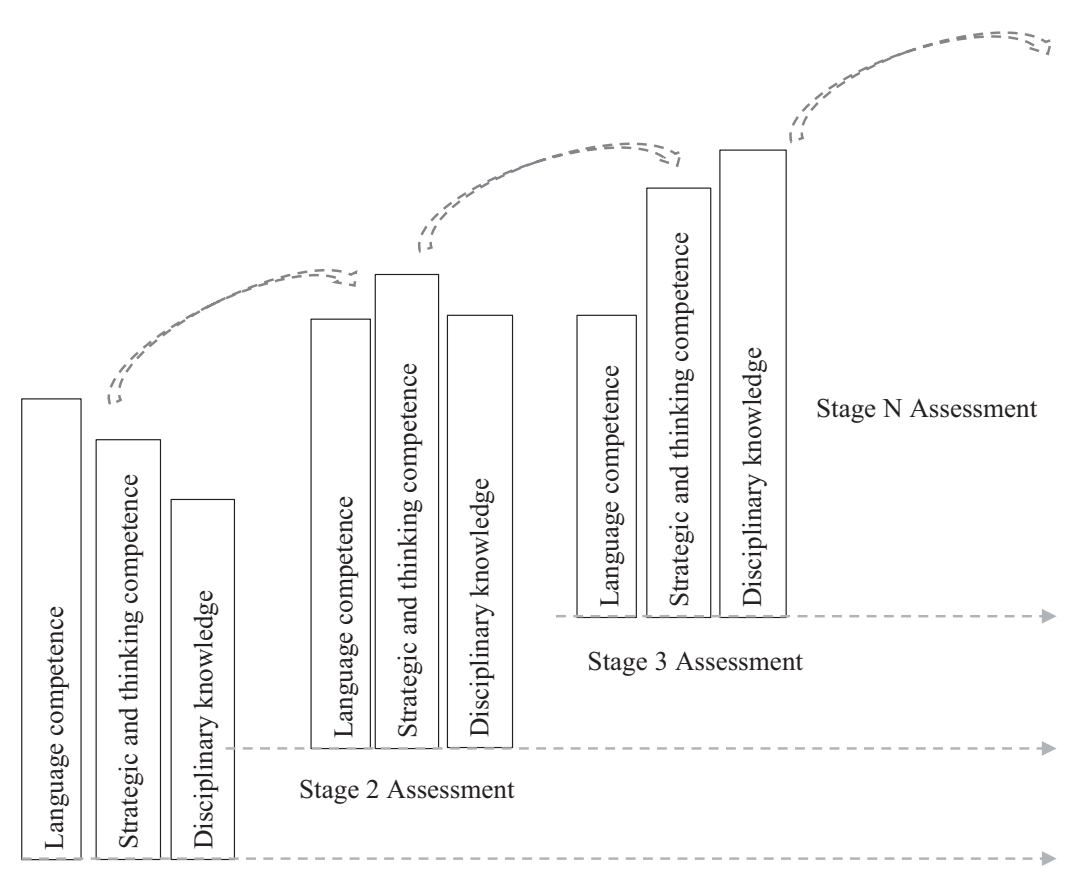

Stage 1 Assessment

Figure 8.1 Progressive assessment of the DLAS.

put, construct-representation based validation study is about additive effects of construct-relevant components, whereas componential validity for DLAS starts from this construct-representation procedure and moves toward a more comprehensive examination of the complex interaction among these key components. Another difference is that construct-representation based validation study focuses on the relative importance of different constructrelevant components, whereas DLAS componential validity focuses on the participating and non-participating of the theoretically valid components across different stages of learning and performance. For instance, the skill of deduction, as theorized into the component of thinking competence, may not be assessed in English for young learners. This, however, should not lead to the conclusion that the test is not a valid DLAS assessment. Rather, this absence could be regarded as a valid absence (non-participating) more consistent with the educational reality (usually deducing is developed as children become more mature). Indeed, it is not rare to see that language assessment programs based on a conventional view of language ability also choose to 
design such types of absence in their assessment programs, but this absence is rarely officially claimed.

According to ST theories, interaction refers to the interdependence between different factors functioning under a language performance. Interaction validity in line with DLAS can be categorized using different criteria. Using a physical criterion, interaction can be divided into two types of validity: internal interaction validity and external interaction validity. The first refers to the interaction among core components of DLAS: language competence, disciplinary knowledge, and strategic and thinking competence, whereas external validity deals with the dependence of the core of the DLAS with contextual factors located within the individual (e.g., students' motivations, psychical factors, gender) or in broader levels (e.g., social economic status, teacher factors, school policy, public media, ideology, and so forth).

The function of interaction can also be divided into moderation and mediation. Moderation refers to the constraining or amplifying effect of one factor on the relation of another factor to a third factor. Indeed, this idea of moderation is not new. In conventional language validation studies, it has long been found that the effect of background knowledge and strategic competence on language performance varies with the learners' general language proficiency (Cai, 2018; Clapham, 1996; Krekeler, 2006). Most recently, Cai and Kunnan (2019, 2020) identified the model which they called the Island Ridge Curve (IRC). According to this model, the effect of strategic competence and disciplinary knowledge on LSP reading performance fluctuates with the continuous increase in general language proficiency.

Another type of interaction is mediation, or the transmission of effect from one factor to another (MacKinnon et al., 2007). Just like the idea of moderation, the concept of mediation is also not new to DLAS. In their argumentation for the interaction mechanism underlying the CLA, Bachman and Palmer (Bachman \& Palmer, 1996; Bachman \& Palmer, 2010) claimed that strategic competence functions as the mediator between language competence and the outer language performance. Regardless, in the language assessment literature, to the authors' best knowledge, systematic investigation of this mediation effect is scant. An exception is the study by Cai (2020). Cai systematically studied the interactions between background knowledge, strategic competence, and language knowledge in determining an LSP reading performance. By concluding his findings with a Triple-Decker Model, the author found that it was internalized disciplinary knowledge and language knowledge that mediates the effect of strategic competence on language performance. However, Cai's conclusion was only based on cross-sectional data rather than on a longitudinal observation of the dynamics of the mediation effect. Regardless, the mechanism of mediation has emerged as an important aspect to be investigated for validation studies based on the DLAS.

The intention of progressive validity is to link the evolution of componential participation with the progress of EMI disciplinary studies. Demands 
on English language, thinking competence, and disciplinary knowledge are likely to vary during different stages of EMI studies (Cai \& Cheung, 2019). It is possible that, during early stages of EMI studies, students are confronted to similar extents with challenges from discipline-related language competence, new disciplinary knowledge and thinking with these new disciplinary knowledges. However, as students' disciplinary and language competences accumulate simultaneously, their application of thinking competence will become more ready for knowledge construction. As this thinking competence is more ready for use, students' acquisition of discipline-related language competence and knowledge construction becomes much easier. In this sense, the development of language competence, disciplinary knowledge, and thinking competence may display different speeds of development during different stages of EMI studies. In a study set in Hong Kong, Cai and Cheung (2019) found emphasis of EMI writing assignments on argumentative essay writing decreased during the whole span of EMI studies, whereas a reversed trend was found with descriptive writing. A similar trend was identified by Gardner and Nesi (2012) with EFL learners in British universities. Although from two different perspectives (one from curriculum materials and another from learners' responses), the two exemplified studies produced the same information: demands of EMI studies on language competence, disciplinary knowledge, and thinking competence vary across the span of EMI studies and very likely, the development of students' disciplinary knowledge, language competence, and thinking competence vary either in parallel, or interactively. Assessment programs developed based on DLAS need to cater to these parallel progressions of the core DLAS components and the interaction among these progressions across repeated assessments.

Bipolarity validity refers to validation of the assumption that the effect of one participating component on the proximal factor may switch between being positive and being negative. Traditional validity studies usually assume that the potential effect of a participating factor is either positive (e.g., language competence) or negative (e.g., anxiety). Drawing on this validity assumption, students engaging more in thinking during their performance on EMI tasks should benefit more from their thinking than students engaging less when solving EMI knowledge construction tasks. Similarly, academic motivation (e.g., self-efficacy) is usually found to benefit students' learning (Schunk \& Zimmerman, 2007) whereas test anxiety is found to be harmful for student learning (von der Embse et al., 2018). However, thoughts from ST as well as from recent development in educational psychology and language testing research suggest that this assumption of monotonous potential may be problematic with these affective and cognitive factors. The idea of bipolarity is prompted by the "golden mean" philosophy of Aristotle more than two millennia ago. The theory proposed that excellence (virtuous disposition) lies in a middle point between two extremes (Bartlett \& Collins, 2011). Put another way, the ideal value along a trait continuum should not be too 
little, nor too much. To take this thought a little further, when the value of a trait moves from the lower extreme toward the middle (the "golden mean"), the potential contribution of the participating factor may show a positive motion (i.e., more is more); however, when the value of the trait passes the golden mean, the motion of the effect may turn downward (i.e., more is less). This bipolarity of psychological factors is illuminating an increasing number of scholars in psychology and educational psychology (Niemiec, 2019; Samuel \& Tay, 2018). Coincidently, the bipolarity of participating components also corresponds with the nonlinear effects of cognitive factors such as learner strategies (Hong-Nam \& Leavell, 2006; Hong-Nam et al., 2014) and background knowledge (Clapham, 1996) on language performance. Most recently, Cai and Kunnan $(2019,2020)$ summarized the nonlinear function of these cognitive factors on LSP performance in the Island Ridge Curve (IRC). According to the IRC, the potential of strategy use and disciplinary knowledge effect may exhibit two opposite directions: a downward potential and an upward potential. The upward potential mostly occurs with students with language competence approaching the peak (sort of a "golden mean") while the downward potential mostly occurs in students with language competence moving above the peak. Polarity validity studies aligned with the DLAS need to explore whether the polarity mechanism exists with these DLAS components at more subtle levels and with contextual factors such as academic motivation factors (e.g., language self-concept, self-efficacy, master goals, performance goals, interest, utility value of language studies, and so forth).

\section{Integrating DLAS into EMI programs}

Although discussions on ST and language as a dynamic system prevail in the literature, there is no consensus on the best practices for integrating this innovative view of language into language instruction for EMI programs. The DLAS provides a dynamic view of the core competences involved for EMI students to accomplish knowledge construction using English language and of the functional mechanism to accomplish them.

\section{EMI language curriculum}

EMI language enhancement needs to have a curriculum based on a needs analysis of the EMI studies. For such a needs analysis, it is not enough to focus on the linguistic knowledge and language skills as language researchers usually do (Brown, 2016); one has to look into the contents of the EMI curriculum and course outlines, for requirements on language, disciplinary knowledge, and levels of thinking (e.g., Cai \& Cheung, 2019). When developing the curriculum for a language enhancement program for EMI students, it must be highlighted that the goals of the language program are to enhance students' language readiness so that they can effectively engage in the process of 
knowledge construction and to reduce their cognitive load due to language barriers. Although the language curriculum of EMI programs bears similarity with a conventional language curriculum in its attention to linguistic knowledge, the EMI language curriculum needs to focus more on the elements of disciplinary knowledge and thinking.

To ensure effective EMI, the language enhancement curriculum needs to reflect the progression of different types of disciplinary knowledge across different stages of EMI studies. Duffy (2014) defines disciplinary knowledge as "knowledge drawn from the unprecedentedly complex and prolific bodies of discourse built up around newly configured professions and disciplines" (p. 2). Cai and Kunnan (2018) further distinguished between domain-specific disciplinary content knowledge that emphasizes the knowledge specific to a subdiscipline (e.g., paediatrics knowledge) and domain-general disciplinary knowledge (e.g., general medical knowledge that every medical professional needs to master). To reflect this disciplinary feature, language curriculum development needs to cater to language features particular to that discipline at various levels (Hyland, 2012; Hyland \& Bondi, 2006): the lexical level (e.g., developing discipline vocabulary lists, on top of the academic vocabulary list), the syntactical level (e.g., identifying sentence patterns particular to the field), and the textual level (e.g., identifying particular text types appropriate for the disciplines and stages of studies).

More importantly, in EMI disciplinary studies, it is common to find that courses covering domain-general disciplinary knowledge are offered during early stages while more domain-specific courses are offered during later stages (Cai \& Cheung, 2019). This progressive feature needs to be reflected in the design of the language curriculum. By nature, disciplinary knowledge differs between declarative knowledge (which is further distinguished into factual and conceptual knowledge) and procedural knowledge (Anderson et al., 2001; Bloom et al., 1956). Given this difference, the demands of mastery of different types of knowledge on learners' cognitive competence also vary (Bloom et al., 1956). The complex nature of this interaction among linguistic factors, disciplinary knowledge, thinking competence, and the progressive features of the interaction needs to be reflected in language enhancement curriculum. A good example of EMI curriculum development is Cai and Cheung (2019). In their analysis of EMI writing assignments, the authors not only examined the componentiality of EMI writing assignment requirements, but also the transition of these components across different stages of EMI studies. Lo and Fung (2020) identified similar progressive patterns in secondary school students.

\section{EMI instruction pedagogy}

The goal of education systems is to foster an environment where teachers and students share in the responsibility for creating the curriculum and pedagogy which enables the co-construction of knowledge (Spain, 2019). The DLAS 
provides a holistic perspective for teachers and learners to understand the objectives of learning and the potential paths leading to this successful collaboration. The DLAS encourages an expansionist view of considering multiple perspectives to seek answers and solve real problems. This approach accommodates the complex organization of different cognitive functions involving linguistic competence, disciplinary knowledge, and thinking competence, which promotes transdisciplinary professionalship for sustainable and adaptable learning (Spain, 2019). In line with DLAS, language and subject teachers serving EMI programs should facilitate learning by transcending pedagogical boundaries and linking their instruction more closely to students' ecological settings (Robinson, 2009).

Language teachers need to recognize all requirements essential for their students to thrive, not merely survive, in their EMI programs. This necessitates the language teachers to not only determine different perspectives that their language courses need to emphasize and the extents of that emphasis, but also to develop the disposition to seek collaboration with colleagues in relevant disciplinary areas, or from their own students who might be more familiar with the content that the language course is dealing with. They need to decide, on the condition that all aspects of DLAS components need to be accommodated, the extents to which different aspects of the DLAS components are emphasized during different stages of EMI studies. To achieve this goal, EMI language courses may be co-taught by language specialists together with a disciplinary teacher, wherever resources allow for such a practice, or by inviting other disciplinary teachers to join the class at appropriate times.

Alternatively, language instructors can organize activities to help students to activate their disciplinary knowledge and thinking skills before they proceed with their language tasks. During language task performance, the language instructors' major duties include not only helping students to identify discipline-related linguistic patterns (Hyland \& Tse, 2009), but more importantly, helping them to foster learning capacity in using disciplinary knowledge, linguistic competence, and thinking skills to thrive in knowledge construction. This emphasis of DLAS-oriented pedagogy is similar to the idea of disciplinary literacy (Shanahan \& Shanahan, 2008) in the general area of educational studies.

Subject teaching in line with the DLAS also accommodates the complex organization of linguistic knowledge, disciplinary knowledge, and thinking competence, as well as the dynamic evolution of this interaction. Different from EMI language programs, however, in regular knowledge construction classes only limited attention needs to be given to linguistic issues, unless teachers find their students' language proficiency becomes such a concern that it makes knowledge construction difficult, or when certain language skills are in special need during a particular time of study. For instance, projectbased academic writing is usually an important constituent of EMI students' Honors' Project. For this requirement, students need to go through the whole 
process of identifying a problem, reviewing the literature, designing the study, collecting the data, conducting analysis, and writing up the findings. This whole scholarly process is unique in that it requires specialized knowledge and skills in integrating language competence, disciplinary knowledge (declarative and procedural), and thinking competence. In this case, a language specialist highly knowledgeable in the relevant disciplinary knowledge, or a subject specialist highly competent in English language may be appropriate for providing intensive training for students with such a need.

A final aspect related to the integration of DLAS to EMI instruction are course assignment tasks. DLAS in course assignments is reflected in two ways: descriptions of course assignments and rubrics for evaluating students' work. The descriptions for the course assignments need to explicate the purpose of the task and the aspects of evidence expected to be extracted from students' performance. For a language course, the assignments need to specify what kinds of language skill(s) the tasks are expected to elicit from students' performances, the types of disciplinary knowledge (domain-general or domain-specific, declarative or procedural) expected of the students to employ, the extents to which such types of knowledge are evaluated (i.e., awareness or application), and the types of and extents to which different levels of thinking skills are required. More importantly, it is necessary to provide transparent marking rubrics with analytical criteria, each aligned with the core components of the DLAS. Besides, a score weighting scheme needs to be provided to reflect the progressive emphasis on different aspects of the DLAS during different stages of EMI studies.

An important note for course assignments in language-oriented courses is that, for the evaluation of performance-based assignments, content is often provided as a criterion together with fluency and language form in analytical rubrics, for example, the Jacobs' scale (Jacobs et al., 1981). However, there is little literature regarding what this content particularly refers to (Alderson, 2005; Knoch, 2009). In line with the DLAS, content needs to be included as a key criterion for EMI language assignments to refer to the fulfilment of applying relevant disciplinary knowledge for meaningful disciplinary communication. The weighting of this criterion to the overall score needs to be aligned with the dynamic view by considering the developmental trajectories of different aspects of the DLAS across different stages of studies. Quite similar to language-oriented courses, discipline-based courses usually describe requirements for disciplinary knowledge and, most of the time, the thinking skills required for accomplishing the tasks, but leaving descriptions of language demands overlooked. This overlooking of language elements is reflected in marking rubrics as well. It is not clear, however, whether and to what extents teachers' final marking is affected by their impressions of students' language presentation embedded in their assignment responses. To reduce inaccuracies possibly contained in final marking, it is highly necessary for EMI subject course descriptions to make transparent the criteria used to mark student performance and the weighing involved with each criterion. 
This weighing scheme must not be static but apply a developmental view across the whole span of EMI studies.

\section{Teacher training}

Teachers' professional practices may vary depending on their inherent epistemological beliefs (Dafouz \& Smit, 2014). Before teachers can engage in instruction activities that can develop DLAS for students, they need to be versed in DLAS themselves. First and foremost, confusion needs to be clarified regarding the goals of EMI programs (i.e., knowledge construction) and the inseparability of language, disciplinary knowledge, and thinking competence, as well as interrelations of these core competences with contextual factors such as student motivation that determine the extents of students' learning engagement. Accordingly, language instructors may fail to understand that disciplinary knowledge, such as domain-general and domain-specific disciplinary knowledge (Cai \& Kunnan, 2018), is an indispensable component for students if they are to engage in meaningful language training. Likewise, subject teachers may fail to understand that deficiency in language competence (e.g., lexical, syntactical, and textual knowledge related to the discipline) compromises the efficiency of knowledge construction if this deficiency cannot be identified and remedied in time. In a similar way, both language teachers and subject teachers may underestimate the importance of thinking competence, which underpins all human cognitive activities (Ennis, 1996).

Teacher development activities can provide both language and subject teachers in their early stages of teaching with the basic tenets of DLAS, as well as training to improve their recognition of the DLAS in their language or subject teaching practice. During this training, subject teachers and language teachers can be invited to elucidate their reflections on their subject teaching and language teaching, respectively, in line with DLAS. In addition, experts in thinking skills can be invited to teach both cohorts of teachers how to apply language skills and world knowledge to develop their own thinking skills as well as how to develop activities that can foster students' development of such higher-order thinking skills for knowledge construction.

\section{Conclusion}

Drawing on existing language ability theory and dynamic Systems Thinking (ST), this chapter proposes the DLAS as a link between language assessment theories and dynamic ST. The DLAS features two subsystems: a space system that accommodates the complex interaction among language competence, disciplinary knowledge, and thinking competence, and a temporal system that caters to the evolving nature of core components and the interaction mechanism. To explicate the features of the DLAS, the authors maintain seven features as the manifestation of the functioning of the DLAS: componentiality, interaction, nonlinearity, parallel pacing, dynamic interdependence, bipolarity, 
and fluctuation. Componentiality recognizes the components that consist of the core of the DLAS. Interaction refers to the temporary interdependence (i.e., constraining, amplifying, and mediating) among these core components with contextual factors located in different levels of the ecological system. Nonlinearity depicts the developmental pattern independent of or dependent on each of the core components and contextual factors. Parallel pacing refers to the idea that each core component has the tendency to develop with its own tempo and magnitude. Dynamic interdependence means that the development of each core component may depend on the development of another component. This is the longitudinal correspondence to the temporal interdependence in a relatively static manner. Bipolarity refers to the opposite directions of a motion potential (e.g., the tendency to increase or to decrease) within the DLAS. Fluctuation suggests that the bipolar motion may switch between moving downward and moving upward. The authors also propose four types of validity for assessment practitioners: componentiality validity, interaction validity, progression validity, and bipolarity validity as aspects for researchers to validate DLAS assessment programs.

In the end of the chapter, the authors propose principles by which DLAS may be integrated into the EMI curriculum through curriculum development, classroom instruction, and teacher development. The authors acknowledge that this framework of DLAS is tentative and needs more intensive follow-ups, both theoretically and empirically. Nevertheless, the DLAS is a first attempt at language assessment, and should at least provide a prompt for future discussion in borrowing thoughts from ST to illuminate endeavors in language assessment against the backdrop of the global movement of EMI.

\section{References}

Ackoff, R. L. (1971). Towards a system of systems concepts. Management Science, $17(11), 661-671$.

Alderson, J. C. (2005). Diagnosing foreign language proficiency: The interface between learning and assessment. London: Continuum.

Anderson, L. W., Krathwohl, D. R., \& Bloom, B. S. (2001). A taxonomy for learning, teaching, and assessing: A revision of Bloom's taxonomy of educational objectives. London: Longman.

Afshar, H. S., \& Movassagh, H. (2017). On the relationship among critical thinking, language learning strategy use and university achievement of Iranian English as a foreign language majors. The Language Learning Journal, 45(3), 382-398.

Bachman, L. F. (1990). Fundamental considerations in language testing. Oxford, UK: Oxford University Press

Bachman, L. F., \& Palmer, A. (1996). Language testing in practice: Designing and developing useful language tests. Oxford, UK: Oxford University Press.

Bachman, L. F., \& Palmer, A. (2010). Language assessment in practice: Developing language assessments and justifying their use in the real world. Oxford, UK: Oxford University Press. 
Bartlett, R. C., \& Collins, S. D. (2011). Aristotle's Nicomachean ethics. Chicago: University of Chicago Press.

Berman, R., \& Cheng, L. (2010). English academic language skills: Perceived difficulties by undergraduate and graduate students, and their academic achievement. Canadian Journal of Applied Linguistics, 4(1), 25-40.

Bloom, B. S., Engelhart, M. D., Furst, E. J., Hill, W. H., \& Krathwohl, D. R. (1956). Taxonomy of educational objectives, handbook I: The cognitive domain. New York: McKay.

Brown, J. D. (2016). Introducing needs analysis and English for specific purposes. New York: Routledge.

Cai, Y. (2018). Detecting the language threshold(s) constraining the effect of background knowledge on LSP reading performance using multi-layered latent moderation analysis. Paper presented at the 4th International Conference on Language Testing and Assessment, Beijing.

Cai, Y. (2020). Examining the interaction among components of English for specific purposes ability in reading: The triple-decker model. New York: Peter Lang.

Cai, Y., \& Cheung, H. T. (2019). Classifying the writing assessment tasks of English as the medium of instruction programs using latent class analysis. International journal of educational research, 95, 143-152. doi:10.1016/j.ijer.2019.02.003

Cai, Y., \& Kunnan, A. J. (2018). Examining the inseparability of content knowledge from LSP reading ability: An approach combining bifactor-multidimensional item response theory and structural equation modeling. Language Assessment Quarterly, 15(2), 109-129. doi:10.1080/15434303.2018.1451532

Cai, Y., \& Kunnan, A. J. (2019). Detecting the language thresholds of the effect of background knowledge on a language for specific purposes reading performance: A case of the island ridge curve. Journal of English for Academic Purposes, 42, article 100795. doi:10.1016/j.jeap.2019.100795

Cai, Y., \& Kunnan, A. J. (2020). Mapping the fluctuating effect of strategic competence on nursing students' English reading performance: An approach of multilayered moderation analysis. Language Testing, 37(2), 280-304.

Canale, M. (1983). On some dimensions of language proficiency. In J. W. Oiler Jr. (Ed.), Issues in language testing research (pp. 333-342). Rowley, MA: Newbury House Publishers.

Canale, M., \& Swain, M. (1980). Theoretical bases of communicative approaches to second language teaching and testing. Applied Linguistics, 1(1), 1-47.

Capra, F. (1996). The web of life: A new synthesis of mind and matter. New York: Doubleday-Anchor Book.

Capra, F., \& Luisi, P. L. (2014). The systems view of life: A unifying vision. Cambridge, UK: Cambridge University Press.

Clapham, C. (1996). The development of IELTS: A study of the effect of background on reading comprehension. Cambridge, UK: Cambridge University Press.

Dafouz, E., \& Guerrini, M. C. (2009). CLIL across educational levels: Experiences from primary, secondary and tertiary contexts. Madrid, Spain: Richmond Publishing.

Dafouz, E., \& Smit, U. (2014). Towards a dynamic conceptual framework for Englishmedium education in multilingual university settings. Applied Linguistics, 37(3), 397-415.

Deane, P., Odendahl, N., Quinlan, T., Fowles, M., Welsh, C., \& Bivens-Tatum, J. (2008). Cognitive models of writing: Writing proficiency as a complex integrated 
skill (ETS Research Report No. RR-08-55). Retrieved from www.ets.org/Media/ Research/pdf/RR-08-55.pdf

Doiz, A., Lasagabaster, D., \& Sierra, J. (2012). English-medium instruction at universities: Global challenges. Bristol, UK: Multilingual matters.

Doiz, A., Lasagabaster, D., \& Sierra, J. (2013). Globalisation, internationalisation, multilingualism and linguistic strains in higher education. Studies in Higher Education, 38(9), 1407-1421.

Douglas, D. (2000). Assessing language for specific purposes. Cambridge, UK: Cambridge University Press.

Duffy, L. (2014). Flaubert, Zola, and the incorporation of disciplinary knowledge. New York: Springer.

Ennis, R. H. (1996). Critical thinking dispositions: Their nature and assessability. Informal Logic, 18(2), 165-182.

Evans, S., \& Green, C. (2007). Why EAP is necessary: A survey of Hong Kong tertiary students. Journal of English for Academic Purposes, 6(1), 3-17.

Evans, S., \& Morrison, B. (2017). English-medium instruction in Hong Kong: illuminating a grey area in school policies and classroom practices. Current Issues in Language Planning, 18(3), 303-322.

Evans, S., \& Morrison, B. (2018). Adjusting to higher education in Hong Kong: The influence of school medium of instruction. International Journal of Bilingual Education and Bilingualism, 21(8), 1016-1029.

Fischer, K. W., \& Yan, Z. (2002). The development of dynamic skill theory. In R. Lickliter \& D. Lewkowicz (Eds.), Conceptions of development: Lessons from the laboratory (pp. 279-312). Hove, UK: Psychology Press.

Floyd, C. B. (2011). Critical thinking in a second language. Higher Education Research \& Development, 30(3), 289-302.

Gardner, S., \& Nesi, H. (2012). A classification of genre families in university student writing. Applied Linguistics, 34(1), 25-52.

Graddol, D. (2006). English next. London: British Council.

Hambleton, R. K., \& Pitoniak, M. J. (2006). Setting performance standards. Educational Measurement, 4, 433-470.

Han, Z. (2019). Profiling learner language as a dynamic system. Bristol, UK: Multilingual Matters.

Hong-Nam, K., \& Leavell, A. G. (2006). Language learning strategy use of ESL students in an intensive English learning context. System, 34(3), 399-415. doi:10.1016/j.system.2006.02.002

Hong-Nam, K., Leavell, A. G., \& Maher, S. (2014). The relationships among reported strategy use, metacognitive awareness, and reading achievement of high school students. Reading Psychology, 35(8), 762-790.

Hu, G., \& Lei, J. (2014). English-medium instruction in Chinese higher education: A case study. Higher Education, 67(5), 551-567.

Hughes, G. (2011). Towards a personal best: A case for introducing ipsative assessment in higher education. Studies in Higher Education, 36(3), 353-367.

Hughes, G. (2014). Ipsative assessment: Motivation through marking progress. New York: Springer.

Hughes, R. (2008). Internationalisation of higher education and language policy. Higher Education Management and Policy, 20(1), 1-18.

Hyland, K. (2012). Discipline and divergence: Evidence of specificity in EAP. English for specific academic purposes: Proceedings of the 2009 BALEAP conference. Reading, UK: Garnet Publishing. 
Hyland, K., \& Bondi, M. (2006). Academic discourse across disciplines. Frankfurt am Main, Germany: Peter Lang.

Hyland, K., \& Tse, P. (2009). Academic lexis and disciplinary practice: Corpus evidence for specificity. International Journal of English Studies, 9(2), 111-129.

Hymes, D. (1972). On communicative competence. In J. Pride \& J. Holmes (Eds.), Sociolinguistics (pp. 269-293). Harmondsworth, UK: Penguin.

Jacobs, H., Zinkgraf, S., Wormuth, D., Hartfiel, V., \& Hughey, J. (1981). Testing ESL composition: A practical approach. Rowley, MA: Newbury House.

Jacobson, M. J., Kapur, M., \& Reimann, P. (2016). Conceptualizing debates in learning and educational research: Toward a complex systems conceptual framework of learning. Educational psychologist, 51(2), 210-218.

Jacobson, M. J., Levin, J. A., \& Kapur, M. (2019). Education as a complex system: Conceptual and methodological implications. Educational Researcher, $48(2), 112-119$.

Jenkins, J. (2014). Global Englishes: A resource book for students. New York: Routledge.

Knoch, U. (2009). Diagnostic writing assessment: The development and validation of a rating scale. Frankfurt am Main, Germany: Peter Lang.

Krekeler, C. (2006). Language for special academic purposes (LSAP) testing: The effect of background knowledge revisited. Language Testing, 23(1), 99-130.

Larsen-Freeman, D. (2012). Complex, dynamic systems: A new transdisciplinary theme for applied linguistics? Language Teaching, 45(2), 202-214.

Larsen-Freeman, D. (2019). On language learner agency: A complex dynamic systems theory perspective. The Modern Language Journal, 103(19), 61-79.

Larsen-Freeman, D., \& Cameron, L. (2008). Complex systems and applied linguistics. Oxford, UK: Oxford University Press.

Lo, Y. Y., \& Fung, D. (2020). Assessments in CLIL: The interplay between cognitive and linguistic demands and their progression in secondary education. International Journal of Bilingual Education and Bilingualism, 23(10), 1192-1210.

MacKinnon, D. P., Fairchild, A. J., \& Fritz, M. S. (2007). Mediation analysis. Annual Review of Psychology, 58, 593-614.

Maringe, F., \& Foskett, N. (2010). Globalization and internationalization in higher education: Theoretical, strategic and management perspectives. London: Continuum.

Mele, C., Pels, J., \& Polese, F. (2010). A brief review of systems theories and their managerial applications. Service Science, 2(1-2), 126-135.

Messick, S. (1989). Validity. In R. L. Linn (Ed.), Educational measurement (3rd ed.) (pp. 13-103). New York: ACE and Macmillan Publishing.

Moore, T. J. (2011). Critical thinking and language: The challenge of generic skills and disciplinary discourses. New York: Bloomsbury.

Nesi, H., \& Gardner, S. (2012). Families of genres of assessed writing. In H. Nesi \& S. Gardner (Eds), Genres across the disciplines: Student writing in higher education (pp. 21-56). Cambridge, UK: Cambridge University Press.

Niemiec, R. M. (2019). Finding the golden mean: The overuse, underuse, and optimal use of character strengths. Counselling Psychology Quarterly, 32(3-4), 453-471.

Robinson, K. (2009). The element. Melbourne, Australia: Penguin Books.

Robinson, K. (2015). Creative schools. Melbourne, Australia: Penguin Books.

Sahlberg, P. (2012). Finnish lessons: What can the world learn from educational change in Finland. New York: Teachers College Press.

Samuel, D. B., \& Tay, L. (2018). Aristotle's golden mean and the importance of bipolarity for personality models: A commentary on "Personality traits and 
maladaptivity: Unipolarity versus bipolarity." Journal of Personality, 87(6), 1097-1102.

Schunk, D. H., \& Zimmerman, B. J. (2007). Influencing children's self-efficacy and selfregulation of reading and writing through modeling. Reading \& Writing Quarterly, 23(1), 7-25.

Scriven, M. (1967). The methodology of evaluation. In R. W. Tyler, R. M. Gagne, \& M. Scriven (Eds.), Perspectives on curriculum evaluation (pp. 39-83). Chicago: Rand McNally.

Shanahan, T., \& Shanahan, C. (2008). Teaching disciplinary literacy to adolescents: Rethinking content-area literacy. Harvard Educational Review, 78(1), 40-59.

Spain, S. (2019). Systems thinking applied to curriculum and pedagogy: A review of the literature. Curriculum Perspectives, 39(2), 135-145.

Toh, G. (2016). Trouble for EMI and EAP (under a new dean). In G. Toh (Ed.), English as Medium of Instruction in Japanese Higher Education (pp. 175-194). New York: Springer.

von der Embse, N., Jester, D., Roy, D., \& Post, J. (2018). Test anxiety effects, predictors, and correlates: A 30-year meta-analytic review. Journal of Affective Disorders, 227, 483-493.

Wächter, B., \& Maiworm, F. (2014). English-taught programmes in European higher education: The state of play in 2014. Bonn, Germany: Lemmens Medien GmbH.

Wolf-Branigin, M. (2013). Using complexity theory for research and program evaluation. Oxford, UK: Oxford University Press. 